ARTICLE

DOI: $10.1038 /$ s41467-018-07572-5

\title{
Artificial optic-neural synapse for colored and color-mixed pattern recognition
}

Seunghwan Seo', Seo-Hyeon Jo', Sungho Kim¹, Jaewoo Shim, Seyong Oh¹, Jeong-Hoon Kim¹, Keun Heo', Jae-Woong Choi ${ }^{2}$, Changhwan Choi (ib ${ }^{3}$, Saeroonter Oh (1) ${ }^{4}$, Duygu Kuzum ${ }^{5}$, H.-S. Philip Wong ${ }^{6}$ \& Jin-Hong Park ${ }^{1,2,6}$

The priority of synaptic device researches has been given to prove the device potential for the emulation of synaptic dynamics and not to functionalize further synaptic devices for more complex learning. Here, we demonstrate an optic-neural synaptic device by implementing synaptic and optical-sensing functions together on $h-\mathrm{BN} / \mathrm{WS} \mathrm{S}_{2}$ heterostructure. This device mimics the colored and color-mixed pattern recognition capabilities of the human vision system when arranged in an optic-neural network. Our synaptic device demonstrates a close to linear weight update trajectory while providing a large number of stable conduction states with less than $1 \%$ variation per state. The device operates with low voltage spikes of $0.3 \mathrm{~V}$ and consumes only $66 \mathrm{fJ}$ per spike. This consequently facilitates the demonstration of accurate and energy efficient colored and color-mixed pattern recognition. The work will be an important step toward neural networks that comprise neural sensing and training functions for more complex pattern recognition.

\footnotetext{
${ }^{1}$ Department of Electrical and Computer Engineering, Sungkyunkwan University, Suwon 16419, Korea. ${ }^{2}$ SKKU Advanced Institute of Nanotechnology (SAINT), Sungkyunkwan University, Suwon 16417, Korea. ${ }^{3}$ Division of Materials Science and Engineering, Hanyang University, Seoul 04763, Korea. ${ }^{4}$ Division of Electrical Engineering, Hanyang University, Ansan 15588, Korea. ${ }^{5}$ Department of Electrical and Computer Engineering, University of California, San Diego, San Diego, CA 92093, USA. ${ }^{6}$ Department of Electrical Engineering, Stanford University, Stanford, CA 94305, USA. These authors contributed equally: Seunghwan Seo, Seo-Hyeon Jo, Sungho Kim. Correspondence and requests for materials should be addressed to J.-H.P. (email: jhpark9@skku.edu)
} 
S ince $\mathrm{Mead}^{1}$ performed the first trial to mimic the biological neural networks (BNNs) of the brain in the 1980s, extensive effort has been made to emulate BNNs by utilizing various synaptic devices ${ }^{2-13}$. In order to properly perform signal processing in BNNs, synaptic plasticity and its timing-dependent learning algorithm are two key computational parameters ${ }^{14}$. However, the scope of initial research on synaptic devices has been mainly focused on simply mimicking synaptic dynamics, such as long-term potentiation/depression (LTP/LTD) ${ }^{15}$, shortterm plasticity ${ }^{16}$, and spike-timing-dependent plasticity (STDP), with filament-forming switching devices ${ }^{2,3}$ or phase change memory devices ${ }^{4}$ in array crossbar structures. Such synaptic devices benefit from superior integration capability and energy efficiency compared to the synaptic devices with lateral transistor structure. However, they suffer from nonlinear potentiation/ depression characteristics ${ }^{17}$, small differences between conduction states ${ }^{18}$, and insufficient conductance states ${ }^{19,20}$. Organic ${ }^{5}$ and carbon nanotube ${ }^{6}$ transistors have recently shown their suitability for emulating of synaptic dynamics with better linear potentiation/depression characteristics and a larger number of usable conductance states ( $>500$ distinct states), in addition to high energy efficiency $(<10 \mathrm{pJ} \text { switching energy })^{21}$.

Beyond this device-level emulation of synaptic dynamics, synaptic devices have also been used to build artificial neural networks (ANNs). Subsequently, pattern recognition tasks ${ }^{2-8}$ have been verified by these ANNs, where winner-take-all ${ }^{6}$ and perceptron networks ${ }^{3}$ are usually applied. However, none of the previous studies have tried to functionalize devices beyond their synaptic functions, for example, by merging them with biometric sensing elements such as vision, auditory, and olfactory sensors. Current works have only demonstrated signal processing in the cerebral cortex with either binary ${ }^{2,3}$ or grayscale MNIST (Modified National Institute of Standards and Technology) datasets ${ }^{6}$. The functional integration of synaptic devices with biometric sensing elements is expected to provide new opportunities for the implementation of neural networks that comprise neural sensing and training functions, thereby enabling power-efficient pattern recognition task for complex (e.g., color-mixed and voice-mixed) patterns. In this article, we demonstrate an optic-neural synaptic (ONS) device that features synaptic and optical-sensing functions. This ONS device was fabricated on a van der Waals (vdW) heterostructure $\left(h-\mathrm{BN} / \mathrm{WSe}_{2}\right)^{22,23}$, which does not have interfacial defects and thereby allows modulating a number of interfacial traps for achieving the synaptic functionalities. Through an opticneural network (ONN) formed by these ONS devices, the colored and color-mixed pattern recognition capability of the human vision system is emulated. In particular, our synaptic device is investigated and compared with other devices reported heretofore, in terms of weight update linearity, number of usable conduction states, stability of each state, and energy efficiency (see Supplementary Table 1).

\section{Results}

$\boldsymbol{h}$-BN/WSe $e_{2}$ optic-neural synaptic device. As shown in Fig. 1, beyond the typical emulation of synaptic dynamics, we have fabricated an ONS device by integrating a synaptic device with an optical-sensing device on the same $h$-BN/WSe $\mathrm{W}_{2}$ heterostructure. The operation of the vdW synaptic device is based on the trapping or de-trapping of electrons in the weight control layer (WCL) on $h$-BN, which modulates the $\mathrm{WSe}_{2}$ channel conductivity (weight of the synapse). The vdW synaptic device will be discussed in detail in the following subsections (see below). Here, the $h$-BN/WSe $e_{2}$ photodetector resistance is modulated as a function of the incident wavelength (see Fig. 1a). The additional analysis on the $h$-BN and $\mathrm{WSe}_{2}$ flakes is described in
Supplementary Figure 1 . The $h$-BN/WSe ${ }_{2}$ interface was confirmed to be clearly formed without polymer residues ${ }^{24}$, owing to our transfer method based on adhesion energy engineering (see Supplementary Figure 2). Because more optical absorption occurs at a shorter wavelength (see Supplementary Figure 3a), a shorter wavelength of light decreases the resistance of the optical-sensing device under a constant drain bias (see Supplementary Figure 3b, c). This reduction in resistance means that more carriers are generated in $\mathrm{WSe}_{2}$ so that the density of carriers trapped in the WCL increases. This subsequently allows the adjustment of the synaptic dynamic properties of the ONS device according to light wavelength conditions. Here, the optical-sensing device was fabricated on the same $h$-BN/WSe ${ }_{2}$ heterostructure where the synaptic device was formed 22,25 . This was done in order for the opticalsensing device to have the proper series resistance, which is comparable to that of the synaptic device, and to enable changing the synaptic properties. If the optical-sensing device has too much larger or smaller resistance value compared to the synaptic device, the resistance control in optical-sensing device by adjusting illumination condition will be ineffective to change the synaptic properties of the ONS device. To identify the synaptic dynamics of the ONS device under different light wavelength conditions, we investigated the device's synaptic plasticity, the postsynaptic current (PSC), and LTP/LTD. The optical-sensing device was exposed to red $(\lambda=655 \mathrm{~nm})$, green $(\lambda=532 \mathrm{~nm})$, and blue $(\lambda=$ $405 \mathrm{~nm}$ ) lights with an optical power of $6 \mathrm{~mW} \mathrm{~cm}^{-2}$, and we then confirmed the PSC characteristics after applying $\pm 1 \mathrm{~V}$ of voltage pulse $\left(V_{\text {pulse }}\right)$ to the synaptic device (see Fig. $1 \mathrm{~b}$ and Supplementary Figure 4 for the measurement set-up). When exposed to a light with shorter wavelength, the magnitude of the synaptic current increased from $1.55 \mathrm{nA}$ to $2.29 \mu \mathrm{A}$ each, where the extracted conductance change $(\Delta G)$ also presented a significant increase from $0.78 \mathrm{nS}$ to $0.74 \mu \mathrm{S}$ by three orders of magnitude. As shown in Fig. 1c, the LTP/LTD curves were distributed in different conductance regions according to the light wavelength, but they maintained the curved shape related to nonlinearity. If the conductance state varies too nonlinearly, similar to a square root function, additional periphery circuits are typically required to manage the wide range of conductance state changes 26,27 . In addition, a nonlinear conductance response imposes difficulty in achieving a sufficient number of usable conductance states because a voltage pulse disables the induction of a sufficiently large conductance difference in the saturated region. Here, we defined effective conductance states as those in which $\Delta G$ exceeds a certain percentage of $G_{\max } / G_{\min }$ (threshold th $_{\Delta G}$ as described in detail in Supplementary Figure 5). Based on threshold ${ }_{\Delta G}$, we excluded the pulse signals that induced states with small $\Delta G$ unimportant for neuromorphic computing, thus reducing power consumption during computation. For the quantitative analysis of the LTP/LTD characteristics of our ONS device, we extracted a nonlinearity $^{28}$ (see Fig. 1d and Supplementary Figure 6) and the number of effective conductance states (see Fig. 1e). For case 1 with a pulse amplitude of $\pm 0.3 \mathrm{~V}$, the nonlinearity was maintained at the levels of 1.5 (potentiation)/1.5 (depression), regardless of the different light conditions, including a no-light condition. In the remaining pulse conditions with pulse amplitudes of $\pm 0.5 \mathrm{~V}$ (case 2 ) and $\pm 1 \mathrm{~V}$ (case 3 ), the nonlinearity (2.6/6 for case 2 and $2.7 / 18$ for case 3 ) was higher than the values of case 1 ; however, they were also independent of the incident light wavelength. Moreover, the number of effective conductance states did not significantly change despite the different light conditions (82-99 for case $1,75-82$ for case 2 , and $74-82$ for case 3 ), where threshold $_{\Delta G}$ was set to $0.3 \%$. Overall, we observed that (i) the conductance ranges in which the LTP/LTD synaptic characteristics were clearly different as a function of the illuminating wavelength; (ii) the LTP/LTD characteristics, such as the linearity 
a

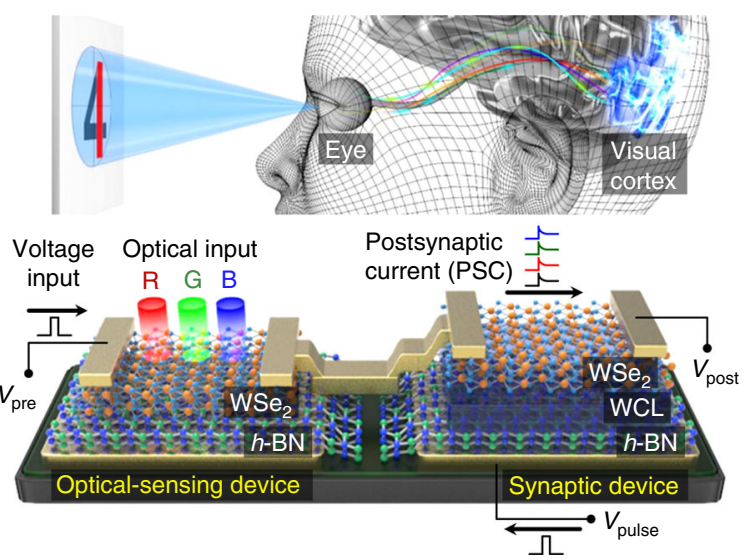

C

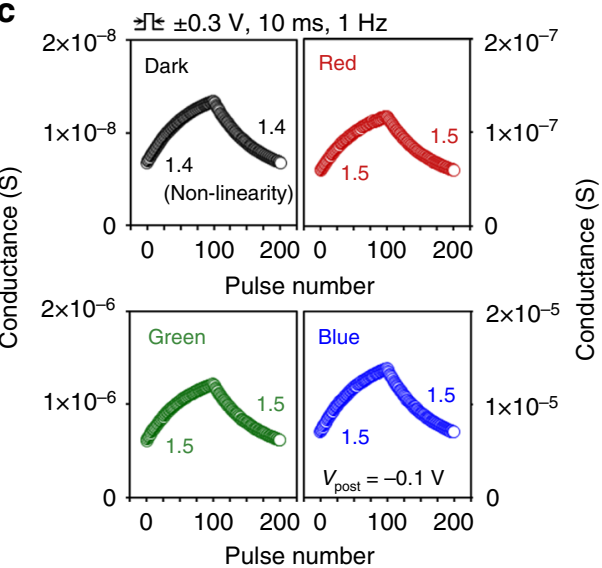

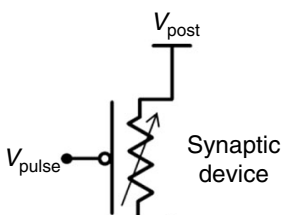

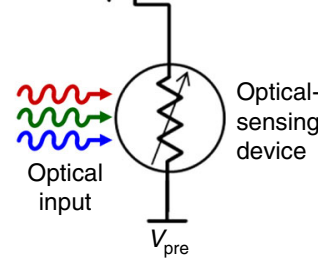

$P_{\mathrm{R}}=P_{\mathrm{G}}=P_{\mathrm{B}}=6 \mathrm{~mW} \mathrm{~cm}{ }^{-2}$

d

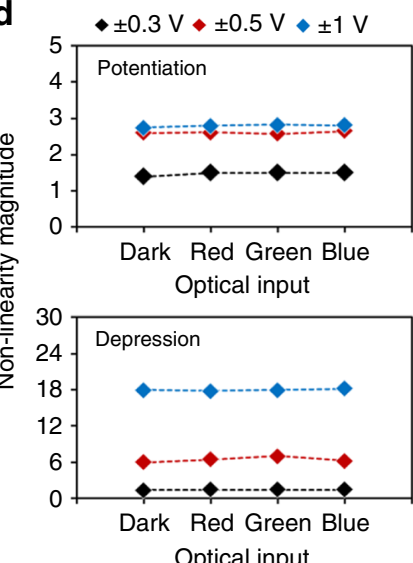

b
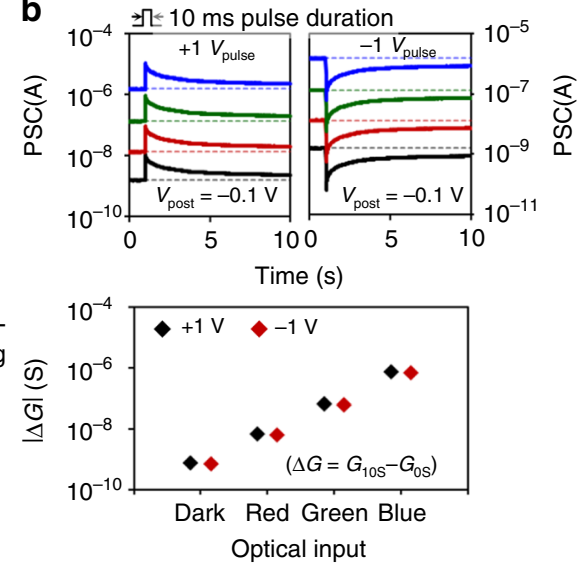

e

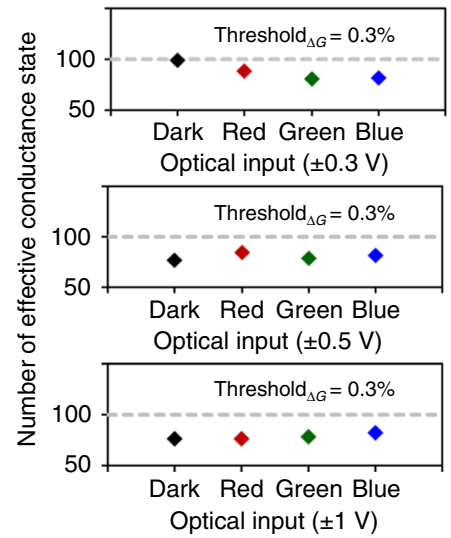

Fig. 1 Integration of the $h$-BN/WSe 2 optic-neural synaptic device. a Schematic of the human optic nerve system, the $h$-BN/WSe $e_{2}$ synaptic device integrated with $h$-BN/WSe 2 photodetector, and the simplified electrical circuit for the ONS device. Here, the light sources were dot lasers with wavelengths of $655 \mathrm{~nm}$ (red), $532 \mathrm{~nm}$ (green), and $405 \mathrm{~nm}$ (blue) with a fixed power density $(P)$ of $6 \mathrm{~mW} \mathrm{~cm}^{-2}$ for all wavelengths. b Excitatory and inhibitory postsynaptic current characteristics and extracted conductance changes of the $h$-BN/WSe $e_{2}$ ONS device under different light conditions (no light and RGB). c Long-term potentiation and depression curves under different light conditions, where the synaptic device is controlled using input pulses with an amplitude of $0.3 \mathrm{~V}$. d, e Nonlinearity magnitude (d) and the number of effective conductance states (threshold ${ }_{\Delta G}=0.3 \%$ ) (e), which were extracted for different wavelengths

and the number of effective conductance states that significantly affect the pattern recognition rate, were almost independent of the light wavelength. Because an optical power is another important information about the optical input, the ONS device eventually needs to be distinctly operated according to the power as well as the wavelength of the optical input. To account for environmental challenges in real-world applications, we exposed our devices to ambient conditions over an extended period of time (see Supplementary Figure 7). Even after 1 month under such conditions, our device proved to be unaffected and worked properly, highlighting their potential for future applications.

Characteristics of $\boldsymbol{h}$-BN/WSe ${ }_{2}$ synaptic device. The synaptic plasticity of the ONS device derives from the operation of the vdW synaptic device with the WCL. The first step toward achieving this synaptic device was to create a charge trapping layer on top of $h$-BN for adjustment of the $\mathrm{WSe}_{2}$ channel conductivity $^{29}$. By forming the WCL on $h-\mathrm{BN}$ with an $\mathrm{O}_{2}$ plasma treatment, we implemented the vdW synaptic device, as shown in Fig. 2a. The hysteresis characteristic was observed in the current between the presynaptic and postsynaptic terminals, which was dependent on the voltage applied to the synaptic cleft terminal $\left(V_{\mathrm{SCT}}\right)$. This occurs because charges trapped in the WCL partially screen $V_{\mathrm{SCT}}$ and thereby influence the current flow through the synaptic device (see Supplementary Figure 8 ). Figure $2 \mathrm{~b}$ shows cross-sectional transmission electron microscopy (X-TEM) images taken near the WCL of the synaptic device. After the $\mathrm{O}_{2}$ plasma treatment was performed for $5 \mathrm{~min}$, an $11.3 \mathrm{~nm}$-thick WCL was observed on the $h$-BN surface region. In addition, as seen in Fig. 2c, high-resolution electron energy loss spectroscopy (EELS) and energy-dispersive X-ray spectroscopy (EDS) mapping analyses were performed to investigate the atomic compositions of the $\mathrm{WSe}_{2} / \mathrm{WCL} / h$ - $\mathrm{BN}$ region. In the WCL, signals related to $\mathrm{O}$ and $\mathrm{B}$ elements clearly appeared, but $\mathrm{N}, \mathrm{W}$, and Se signals were not present, indicating that the WCL consisted of the oxidized boron transformed from $h$-BN. As expected, this WCL was not observed in the EELS and EDS mapping images for the control sample that was not subjected to the $\mathrm{O}_{2}$ plasma treatment (refer to Supplementary Figure 9). To analyze the dynamic nature of the conductivity (see Fig. 2e), voltage pulses with 0.1 and $1 \mathrm{~V}$ amplitudes were applied to the synaptic device. In the case where the voltage pulse was $0.1 \mathrm{~V}$, the transient electrons trapped in fast traps recovered, and the abruptly increased current returned to its initial value after $8 \mathrm{~s}$, resulting in no persistent $\Delta G$. On the other hand, when a pulse with $1 \mathrm{~V}$ amplitude was applied, a positive $\Delta G$ occurred because electrons were captured and held in the traps deeper inside the WCL. Calculation of the trap density in the WCL gave a value of approximately $7.4 \times 10^{-17} \mathrm{~cm}^{-3}$. When 
a

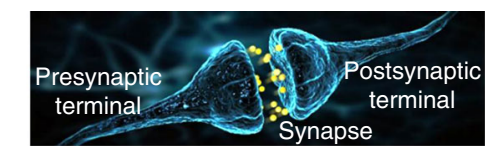

Presynaptic Postsynaptic current Postsynaptic
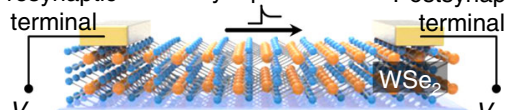

$V_{\text {pre }}$

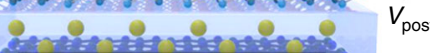

Weight control layer (WCL)
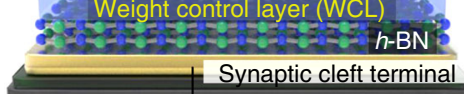

- W $B \quad \stackrel{V_{\text {pulse } \Omega}^{\rightleftarrows}}{\rightleftarrows} V_{\text {SCT }}$

- Se $\mathrm{N}$

e

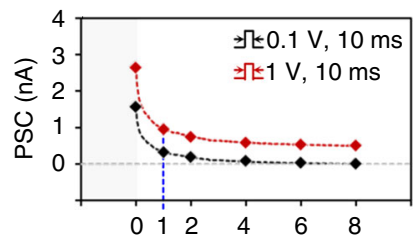

Time after pulse (s)

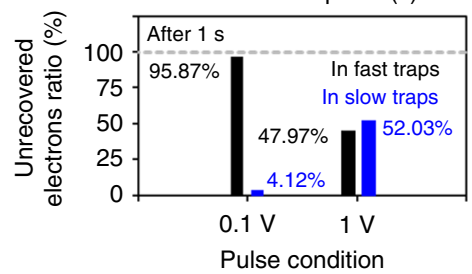

b

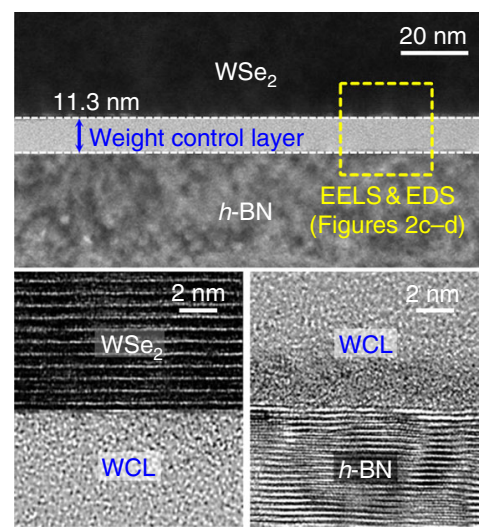

$f_{\text {At } 0}$ s after pulse

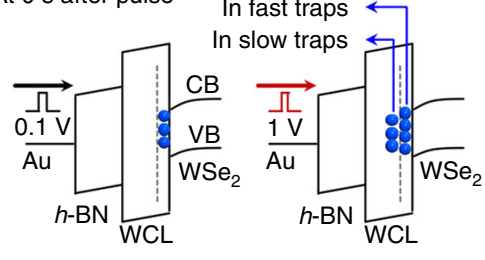

After de-trapping

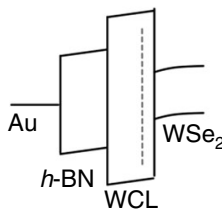

C

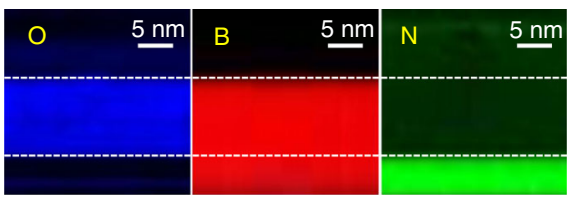

\section{d}

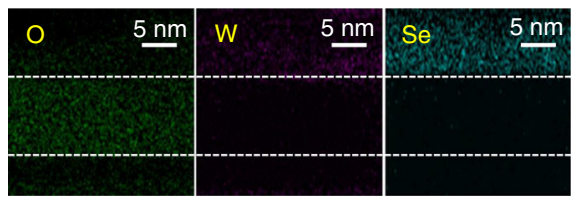

g
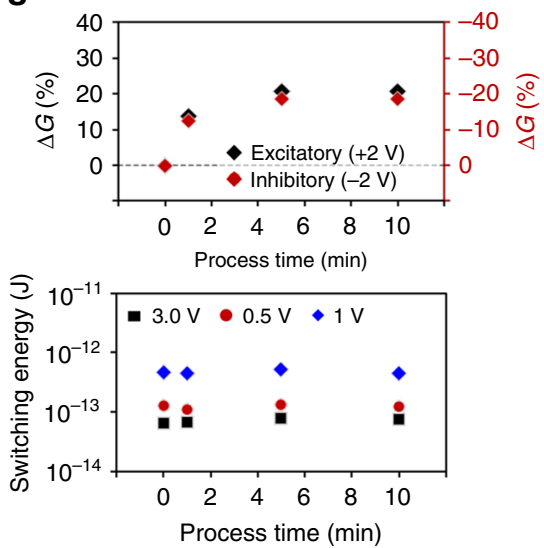

Fig. 2 Structure and operating mechanism of the $h-B N / W e_{2}$ synaptic device. a Functional/structural/architectural comparison of biological synapse with our synthetic WSe $2 / \mathrm{WCL} / h$-BN synaptic device. $\mathbf{b} \mathrm{X}$-TEM image of the WSe $/ \mathrm{WCL} / h$-BN structure, and the high-resolution images corresponding to the WSe $2 / W C L$ and WCL/h-BN interfaces. c, d EELS (c) and EDS (d) mapping images obtained on the cross-section of the WSe $2 /$ WCL/h-BN structure. e Current relaxation curves after pulse amplitudes of $0.1 \mathrm{~V}$ and $1 \mathrm{~V}$, and contribution ratio from unrecovered electrons in fast traps and slow traps at $1 \mathrm{~s}$ after the pulse. $\mathbf{f}$ Illustration of energy band diagrams after pulse and after de-trapping of carriers in fast traps. $\mathbf{g}$ Change in postsynaptic conductance and the switching energy measured as a function of $\mathrm{O}_{2}$ plasma process time. Here, all the $V_{\text {pulse }}$ were applied with a duration of 10 ms

extrapolated to current memory cell sizes, the number of trapped electrons was on the same order of electrons stored on a floating gate of a flash memory cell (see Supplementary Figure 10) (http:// www.itrs2.net/). The Fig. 2e shows the ratio of the remaining charges in fast traps and slow traps at $1 \mathrm{~s}$ after the pulse, according to the pulse amplitude. When a pulse with higher amplitude was applied, the contribution of slow traps to the increase in PSC increased noticeably, consequently exceeding that of fast traps. As shown in Fig. 2f, by applying a pulse with higher amplitude, more charges are expected to be trapped in slow traps that will not recover at room temperature, which subsequently changes the synaptic conductance of $\mathrm{WSe}_{2}$. We also note that the contribution of fast traps to the conductance change diminishes as time elapses after the pulse (see Supplementary Figure 11). The magnitude of $\Delta G$ increased until the $\mathrm{O}_{2}$ process time reached the 5 min point and then saturated beyond that time, where the $|\Delta G|$ values were similar in both excitatory and inhibitory synaptic responses. We also estimated the switching energy of the synaptic device as a function of the $\mathrm{O}_{2}$ plasma process time (see Fig. $2 \mathrm{~g}$ ). Regardless of the process time related to the amount of traps in the WCL, the switching energy was approximately $66 \mathrm{fJ}$ (at $0.3 \mathrm{~V}$ of presynaptic pulse) and it increased to $532 \mathrm{fJ}$ (at $1 \mathrm{~V}$ of pulse), which is comparable to those of previously reported synaptic devices (see Supplementary Table $1 \mathrm{~b}$ ). $\Delta G$ is a function of the amplitude and duration of the presynaptic pulse for the weight update, such that the dissipated energy per event can be determined by $P=I \times V \times t_{\text {duration }}{ }^{5}$.

For neuromorphic computing based on deep neural networks (DNNs) with the well-studied back-propagation learning algorithm $^{30}$, as mentioned above, synaptic devices are required to have the following characteristics: linear conductance responses $17,19,31$, a sufficient number of effective conductance states $^{19,20,31}$, and high stability in each state. Here, we thoroughly investigated the vdW synaptic device to identify various synaptic dynamics including LTP/LTD and STDP. Figure 3a shows the LTP/LTD characteristic curves for the synaptic device according to three pulse conditions: $\pm 0.3 \mathrm{~V}$ (case 1 ), $\pm 0.5 \mathrm{~V}$ (case 2 ), and \pm 1 $\mathrm{V}$ (case 3). When applying a lower amplitude pulse, the conductance was changed more linearly, subsequently presenting a low nonlinearity of 1.4 (case 1). Figure $3 \mathrm{~b}$ shows that 99 (case 1), 75 (case 2), and 74 (case 3) conductance states were usable when threshold ${ }_{\Delta G}$ was set to $0.3 \%$. Additionally, to confirm if this synaptic device can achieve the number of conductance states that were typically demanded for inference of DNNs $(6-8$ bits $=64-256$ states) ${ }^{32,33}$, we applied excitatory and inhibitory pulses 600 times each (see Fig. 3b). As a result, the nonlinearity was approximately 1.4, and the number of effective conductance states was 599 under a $0.3 \%$ threshold $_{\Delta G}$. Each conductance state was very stable, presenting a very small variation of below $1 \%$ even after the state was changed many times by the excitatory and 

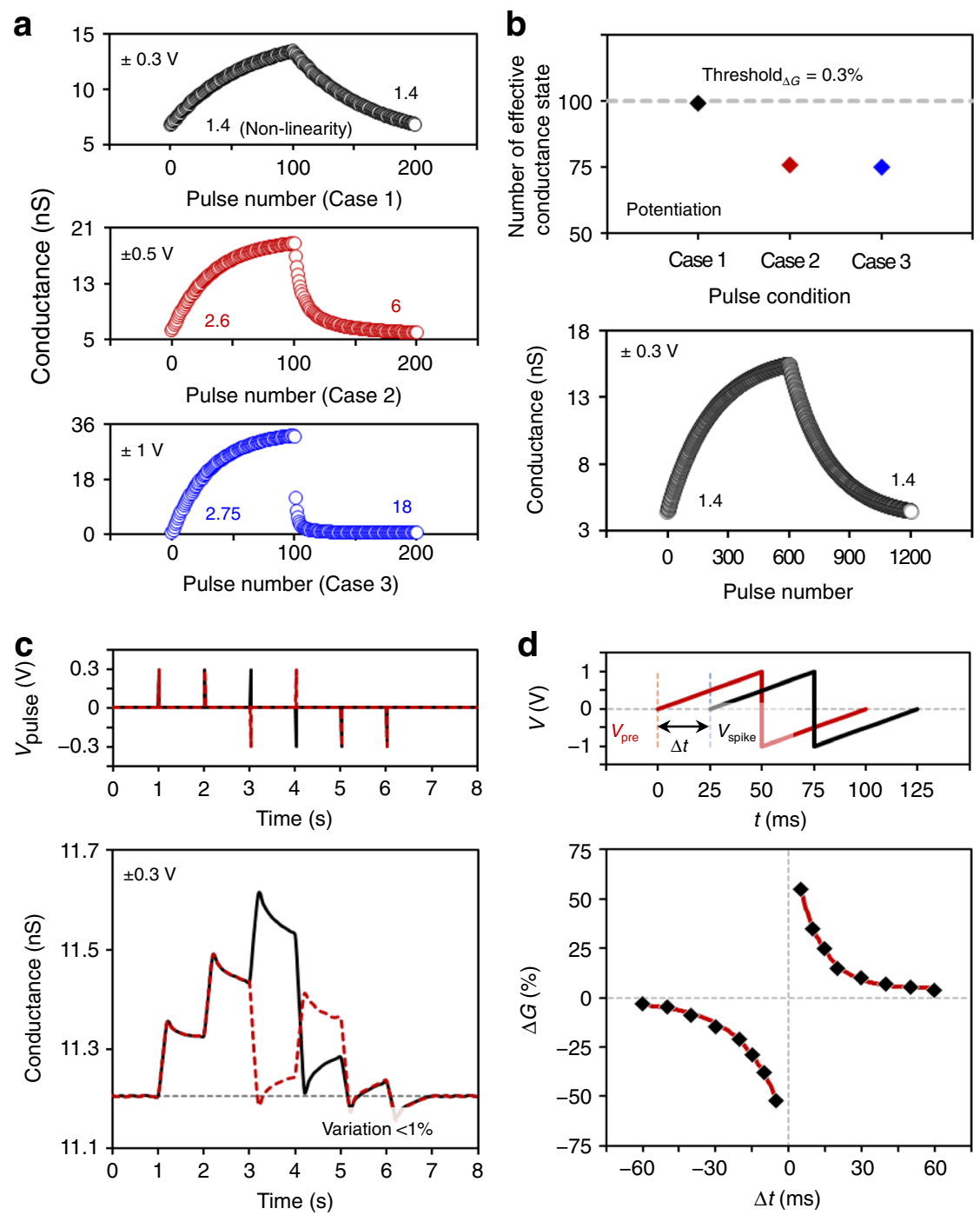

Fig. 3 Synaptic plasticity characterization of $h-\mathrm{BN} / \mathrm{WS} \mathrm{e}_{2}$ synaptic device. a Long-term potentiation and depression characteristics by different input pulses with an amplitude of $0.3 \mathrm{~V}, 0.5 \mathrm{~V}$, or $1 \mathrm{~V}$. b Number of effective conductance states for the three cases with a threshold ${ }_{\Delta G}=0.3 \%$, and LTP/LTD curves when 600 pulses are applied in each potentiation and depression. c Stability of conductance states with below $1 \%$ variation. d Spike-timing-dependent plasticity behavior obtained in the $h$-BN/WSe 2 synaptic device. The pre-spike and post-spike voltages are applied to the presynaptic and SCT, respectively

inhibitory pulses. This can be explained by a similar number of electrons which are trapped and de-trapped in the $h$-BN WCL by applying symmetric positive and negative pulses. In the Supplementary Figure 12, we additionally discuss the long-term variation of conductance states with respect to the various pulse combinations. In Fig. 3d, we also confirm the STDP behavior of our vdW synaptic device, indicating that this device can be applied to spiking neural networks with the STDP learning algorithm ${ }^{14}$. Biological synapses potentiate if a presynaptic spike precedes a postsynaptic spike, and depress if a postsynaptic spike precedes a presynaptic spike ${ }^{34}$. Likewise, in our synaptic device, conductance increased or decreased $(\Delta G / G>0$ or $\Delta G / G<0)$ according to the relative difference between the presynaptic spike $\left(V_{\text {pre }}\right)$ and the SCT spike $\left(V_{\mathrm{SCT}}\right)(\Delta t>0$ or $\Delta t<0)$.

Colored and color-mixed pattern recognition. Following the integration of our synaptic device and optical-sensing device on the $h$-BN/WSe $e_{2}$ heterostructure, we developed an artificial ONN using the extracted device parameters and a simple perceptron network model ${ }^{35}$, finally applying the ONN to the colored and color-mixed pattern recognition tasks. As shown in Fig. 4a, two separate neural networks were developed for recognizing the target color number from the complex mixed-color numerical digits (similar to a color-blindness test). Here, the input layer of the conventional neural network $(\mathrm{NN})$ consisted of the neuron array with the color-filtering function to emulate the biological cone cells of the human vision system, but the synaptic connections were formed without the optical-sensing function. In contrast, in the ONN, an optical-sensing function was added to the synaptic connection. Our artificial cone cell group consisted of 3 neurons, and the groups formed a $28 \times 28$ array, where each cell in the group responded differently to the wavelengths of visible red $(R)$, green $(G)$, and blue (B) light as human eyes do, subsequently inducing different synaptic dynamics. The input signals for both $\mathrm{NN}$ and $\mathrm{ONN}$ were determined as $1 \mathrm{~V}$ (for $\mathrm{R}$ ), $0.5 \mathrm{~V}$ (for $\mathrm{G}$ ), and $0.3 \mathrm{~V}$ (for B) by considering the color. Neurons in each cone cell are fully connected to 2 dedicated classifying neurons (" 1 " and " 4 "), resulting in a connection with 6 classifying neurons at the output layer. In particular, the black weight connections in the NN represent the synaptic devices with the LTP/ LTD characteristic under dark condition, and the connections 
a

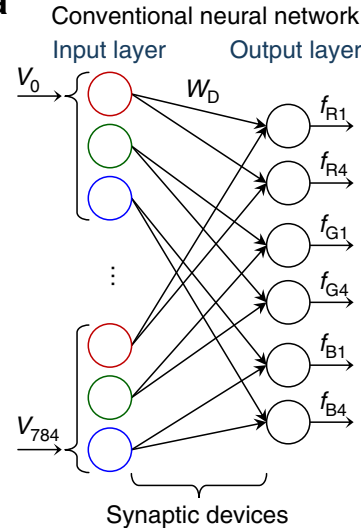

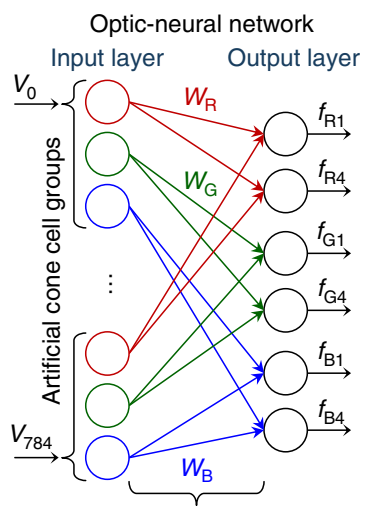

Optic-neural synaptic devices

d

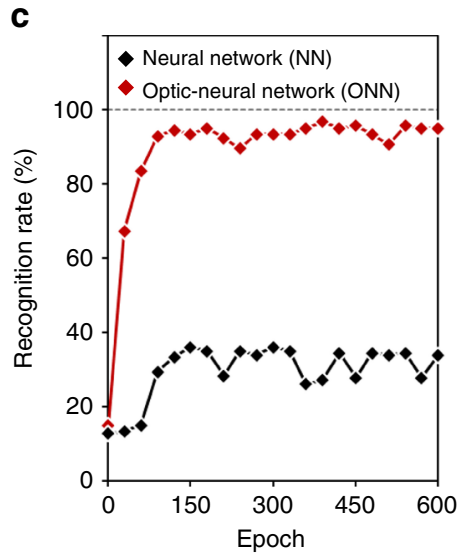

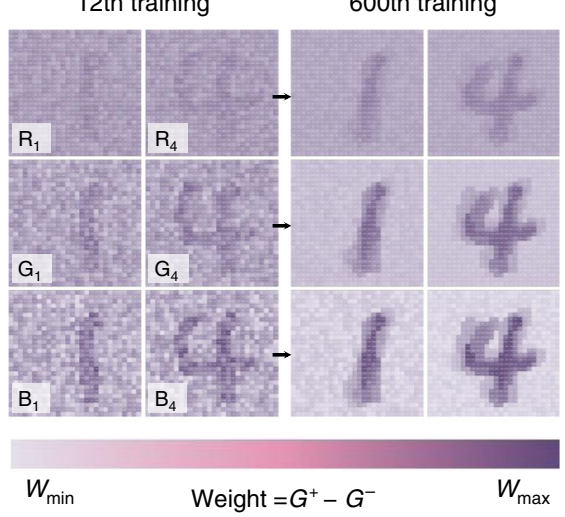

b
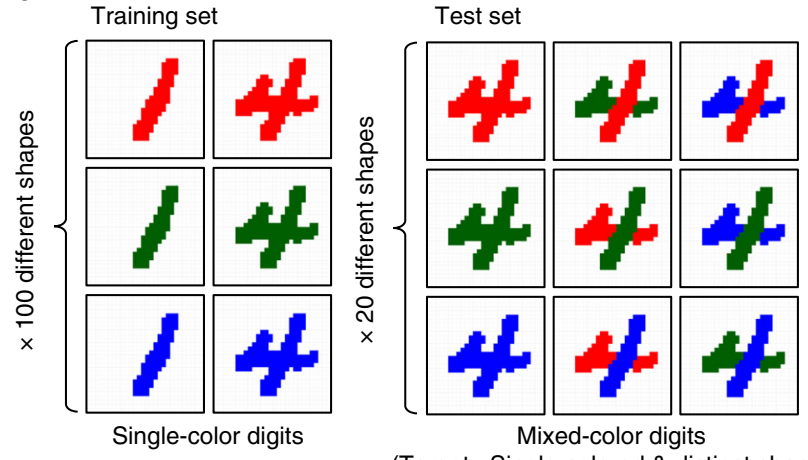

Mixed-color digits

(Target : Single-colored \& distinct shape)

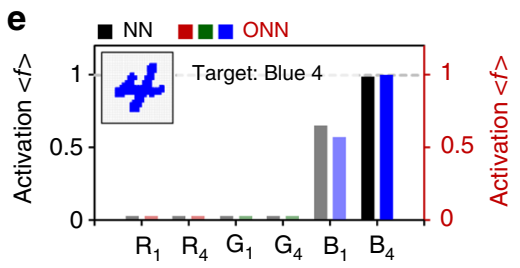

Output neuron

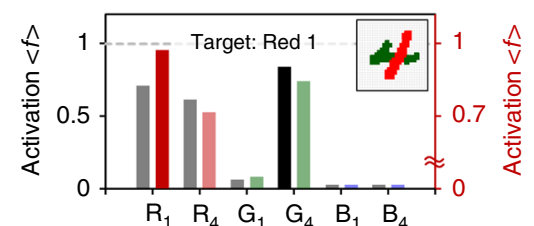

Output neuron

Fig. 4 Colored and color-mixed pattern recognition based on an artificial optic-neural network. a Developed ONN for recognition of $28 \times 28$ RGB-colored images. $\mathbf{b}$ Examples of the training and the testing datasets consisting of single-colored and color-mixed numeric pattern images, respectively. c Recognition rate as a function of number of training epochs. $\mathbf{d}$ Weight mapping images after the 12th and 600th training epoch. e Activation values of each output neuron in cases of a single-colored number (blue 4) and a color-mixed number (red/green-mixed 4) after the 600th training epoch

expressed by RGB (red, green, and blue) color lines in the ONN indicate the synaptic devices with the LTP/LTD characteristics in different $R / G / B$ conductance ranges. The MNIST dataset ${ }^{36}$ was used for the pattern recognition tasks, in which the original image size $(28 \times 28)$ was preserved, but a simple modification was made to generate color-mixed numerical patterns (see Supplementary Figure 13). The objective of this pattern recognition task was to recognize the target numbers from the color-mixed test images (similar to a color-blindness test), where the target numbers are buried in the color-mixed patterns. To train the networks, as seen in Fig. $4 \mathrm{~b}$, we prepared 6 different training datasets with 100 training images for each dataset (600 images in total) for RGBcolored numbers 1 and 4 . We also prepared 9 test datasets with 20 different test images for each dataset (180 images in total) for the pattern recognition test. The 600 different training images were applied to the networks at every training epoch, and we finished the training phase at 600 epochs. At every epoch, the recognition rates for both networks were estimated using 180 test images. The rate for the ONN exceeded $90 \%$ after the 50th epoch and then stabilized (see Fig. 4c). In contrast, the recognition rate for the $\mathrm{NN}$, which was composed of synaptic devices without the opticalsensing function, was below $40 \%$. As the training epochs increased, the synaptic weight values were further optimized for RGB-color-mixed numerical pattern recognition. To confirm this weight optimization, we reconstructed and visualized the synaptic weight values after the 12th and 600th training epochs (see Fig. 4d). The synaptic weights created for the blue patterns became richer than those of the other colors did because the ONS device most strongly responded to blue light input; this indicates that the ONN successfully recognized different-colored numerical patterns (RGB 1 or 4). Finally, the recognition tests for colormixed digit patterns were demonstrated for both networks with the well-trained weight values after the 600th training epoch, and the corresponding results are provided in Fig. 4e. In the case of the blue-colored number 4 , both networks easily succeeded in recognizing the target number by providing the highest activation scores at the B4 classifying (output) neurons. However, for the complex color-mixed number (red/green-mixed 4), the R1 classifying neuron in the ONN produced the highest activation score of 0.98 , while the highest value of 0.83 was obtained from the G4 classifying neuron in the $\mathrm{NN}$.

\section{Discussion}

We demonstrate an ONS device with synaptic and optical-sensing functions by integrating a synaptic device and an optical-sensing device on a vdW heterostructure $\left(h-\mathrm{BN} / \mathrm{WSe}_{2}\right)$. Our ONS device exhibit various synaptic dynamics like LTP, LTD, and STDP according to the light conditions (red $(\lambda=655 \mathrm{~nm})$, green $(\lambda=$ $532 \mathrm{~nm}$ ), and blue $(\lambda=405 \mathrm{~nm})$ ), while maintaining its synaptic plasticity (linearity and number of effective conductance states), where the LTP/LTD characteristics are distributed in different conductance regions. In the $\mathrm{vdW}$ synaptic device, the conductance related to the synaptic weight is adjusted by the charges trapped in the WCL that is formed on $h-\mathrm{BN}$ by $\mathrm{O}_{2}$ plasma treatment. This vdW synaptic device in particular shows good 
linearity with nonlinearity $=1.4 / 1.4$ for weight increase/decrease, a number of effective conductance states about 599 at thresh$\operatorname{old}_{\Delta G}=0.3 \%$, and very small variation below $1 \%$ after the random state changes by excitatory and inhibitory pulses. These excellent synaptic properties clearly highlight the potential of our vdW-based ONS device for building highly accurate neuromorphic ANNs. Following in-depth study and characterization of the vdW ONS device, we develop an ONN that emulates the colored and color-mixed pattern recognition capability of the human vision system. With this ONN we achieve $>90 \%$ recognition rate for color-pattern recognition task, which is similar to a color-blindness test. With this research, where we co-integrate synaptic and biometric sensing elements on the same technology stack, we provide the foundation for future work towards building neural networks that comprise sensing and training functionalities for highly integrated complex pattern recognition tasks.

\section{Methods}

Fabrication and characterization of the synaptic device. The individual control electrodes (line shaped with width of $20 \mu \mathrm{m}$ ) were patterned on the $90 \mathrm{~nm}$-thick $\mathrm{SiO}_{2}$ oxide layer on heavily boron-doped $\mathrm{Si}$ substrate using an optical lithography process, followed by $10 \mathrm{~nm}$-thick Ti and $30 \mathrm{~nm}$-thick Au deposition processes via an e-beam evaporator. The $h$-BN flake was then transferred onto the control electrode by our residue-free transfer method based on adhesion energy engineering (see Supplementary Figure 2). The $\mathrm{O}_{2}$ plasma treatment process was carried out on the $h$-BN flake by a plasma machine (Miniplasma Cube, PLSMART). To stabilize the chamber conditions, $\mathrm{O}_{2}$ gas flowed for $2 \mathrm{~min}$ before the $\mathrm{O}_{2}$ plasma treatment. The treatment conditions are as follows: reactive ion etcher power $(20 \mathrm{~W}, 100 \mathrm{~W})$, plasma pressure $(470 \mathrm{mTorr})$, and $\mathrm{O}_{2}$ flow rate (5 $\mathrm{sccm})$, treatment time $(1,5$, and $10 \mathrm{~min})$. A WSe $\mathrm{W}_{2}$ flake was then transferred onto the WCL/h-BN by using the same transfer method. The postsynaptic and presynaptic electrodes (distance between two electrodes and width of the electrodes were $5 \mu \mathrm{m}$ ) were patterned on the WSe $/ 2 \mathrm{WCL} / h$-BN sample by an optical lithography process, followed by $10 \mathrm{~nm}$-thick Pt and $50 \mathrm{~nm}$-thick Au deposition processes via an e-beam evaporator (see Supplementary Figure 1, optical image of the synaptic device). For the structural and elemental analyses of the $\mathrm{WSe}_{2} / \mathrm{WCL} / h$-BN region, the X-TEM (JEM ARM 200F) and EELS/EDS (GIF quantum ER, $200 \mathrm{keV}$ ) measurements were employed. The fabricated $\mathrm{WSe}_{2} / \mathrm{WCL} / h$-BN synaptic devices were electrically analyzed in terms of current with respect to the control voltage by an HP 4155A semiconductor parameter analyzer.

Fabrication and characterization of the optical-sensing device. The $h$-BN flake was mechanically exfoliated and transferred onto the $90 \mathrm{~nm}$-thick $\mathrm{SiO}_{2}$ layer on heavily boron-doped Si substrate via adhesive tape (224SPV, Nitto). The remaining adhesive tape residue was removed by immersing the samples in an acetone bath for $1 \mathrm{~h}$. The WSe $\mathrm{W}_{2}$ flake was then transferred to the $h-\mathrm{BN} / \mathrm{SiO}_{2} / \mathrm{Si}$ substrate by the residue-free transfer method. The electrodes with $5 \mu \mathrm{m}$ spacing were patterned on the $h-\mathrm{BN} / \mathrm{WSe}_{2}$ sample by an optical lithography process, which were respectively grounded and connected to the presynaptic terminal of the $\mathrm{vdW}$ synaptic device, then followed by $10 \mathrm{~nm}$-thick Pt (for p-type channel) and $50 \mathrm{~nm}$-thick $\mathrm{Au}$ deposition processes via an e-beam evaporator (see Supplementary Figure 1, optical image of the optical-sensing device). The $h$-BN/WSe 2 optical-sensing devices were investigated by electrical measurements (current with respect to control voltage, $I$ $V)$ using an HP 4155A semiconductor parameter analyzer under both dark and illuminated conditions. The light sources were dot lasers with wavelengths of 655 $\mathrm{nm}$ (red), $532 \mathrm{~nm}$ (green), and $405 \mathrm{~nm}$ (blue), and an optical power of $6 \mathrm{~mW} \mathrm{~cm}^{-2}$.

Pattern recognition task. After integrating the synaptic device with the opticalsensing device on the $h-\mathrm{BN} / \mathrm{WSe}_{2}$ heterostructure, in order to confirm the learning capability of our ONS device, we designed neural networks based on a single-layer perceptron model using the extracted ONS device parameters, such as the nonlinearity values (at input signal pulse $= \pm 0.3 \mathrm{~V}$ ) and the number of effective conductance states (at threshold ${ }_{\Delta G}=0.3 \%$ ). We then investigated the recognition rates for the colored MNIST numerical patterns. When the input voltage signals corresponding to the RGB colors (red $=1 \mathrm{~V}$, green $=0.5 \mathrm{~V}$, blue $=0.3 \mathrm{~V}$ ) were applied to the input neurons, currents were obtained at output neurons by a matrix product of the input signal and the synaptic weight $(W)$ values. Here, the synaptic weight is defined by the difference between the conductance values of the two ONS devices $\left(W=G^{+}-G^{-}\right)$. The currents at the output neurons were transformed by a sigmoid activation function, resulting in output neuron signals $(f)$. Based on the delta value $(\delta)$, which is the difference between the output neuron signals $(f)$ and the label values $(k)$ for the input patterns $(\delta=k-f)$, the synaptic weight was determined to be potentiated or depressed. If $\delta>0$ (potentiation phase), then $G^{+}$is increased and $G^{-}$is simultaneously decreased, thereby increasing the synaptic weight $\left(W \uparrow=G^{+} \uparrow-G^{-} \downarrow\right)$. In the depression phase $(\delta<0), G^{+}$and $G^{-}$are decreased and increased, respectively, at the same time $\left(W \downarrow=G^{+} \downarrow-G \uparrow\right)$. These conductance changes $\left(\Delta G^{+}\right.$or $\left.\Delta G^{-}\right)$were determined by the following equations:

$$
\begin{aligned}
& G_{\mathrm{n}+1}=G_{\mathrm{n}}+\Delta G_{\mathrm{P}}=G_{\mathrm{n}}+\alpha_{\mathrm{P}} e^{-\beta_{\mathrm{P}} \frac{G_{\mathrm{n}}-G_{\min }}{G_{\max }-G_{\min }}}\left(\Delta G^{+} \text {or } \Delta G^{-}>0, G^{+} \text {or } G^{-} \uparrow\right) \ldots \\
& G_{\mathrm{n}+1}=G_{\mathrm{n}}+\Delta G_{\mathrm{D}}=G_{\mathrm{n}}-\alpha_{\mathrm{D}} e^{-\beta_{\mathrm{D}} \frac{G_{\max }-G_{\mathrm{n}}}{G_{\max }-G_{\min }}}\left(\Delta G^{+} \text {or } \Delta G^{-}<0, G^{+} \text {or } G^{-} \downarrow\right) \ldots
\end{aligned}
$$

In these equations, $G_{n+1}$ and $G_{n}$ denote the synaptic conductance when the $n+$ 1 th and $n$th pulses are applied, and parameters $\alpha$ and $\beta$ are the conductance change amount and the nonlinearity, respectively (see Supplementary Table 2). The above pattern recognition processing was implemented with MATLAB.

Code availability. Code from this study (MATLAB scripts) is available from the corresponding author upon request.

\section{Data availability}

The data that support the findings of this study are available from the corresponding author upon request.

Received: 8 May 2018 Accepted: 6 November 2018

Published online: 30 November 2018

\section{References}

1. Mead, C. Neuromorphic electronic systems. Proc. IEEE 78, 1629-1636 (1990).

2. $\mathrm{Yu}, \mathrm{S}$. et al. An electronic synapse device based on metal oxide resistive switching memory for neuromorphic computation. IEEE Trans. 8, 2729-2737 (2011).

3. Prezioso, M. et al. Training and operation of an integrated neuromorphic network based on metal-oxide memristors. Nature 521, 61-64 (2015).

4. Wong, H. S. P. et al. Phase change memory. Proc. IEEE 98, 2201-2227 (2010).

5. Burgt, Yvd et al. A non-volatile organic electrochemical device as a lowvoltage artificial synapse for neuromorphic computing. Nat. Mater. 4856, 1-6 (2017)

6. Kim, S., Yoon, J., Kim, H.-D. \& Choi, S.-J. Carbon nanotube synaptic transistor network for pattern recognition. ACS Ami. 7, 25479-25486 (2015).

7. Wang, Z. et al. Memristors with diffusive dynamics as synaptic emulators for neuromorphic computing. Nat. Mater. 16, 101-108 (2017).

8. Choi, S. et al. SiGe epitaxial memory for neuromorphic computing with reproducible high performance based on engineered dislocations. Nat. Mater. 17, 335-340 (2018).

9. Sangwan, V. K. et al. Multi-terminal memtransistors from polycrystalline monolayer molybdenum disulfide. Nature 554, 500-504 (2018).

10. Yoon, S. M., Tokumitsu, E. \& Ishiwara, H. An electrically modifiable synapse array composed of metal-ferroelectric-semiconductor (MFS). IEEE Electron Device Lett. 20, 229-231 (1999).

11. Silver, D. et al. Mastering the game of Go with deep neural networks and tree search. Nature 529, 484-489 (2016).

12. Wang, Z. et al. Fully memristive neural networks for pattern classification with unsupervised learning. Nat. Electron. 1, 137-145 (2018).

13. Sheridan, P. M. et al. Sparse coding with memristor networks. Nat. Nanotech 12, 784-789 (2017)

14. Bi, G.-q \& Poo, M.-m Synaptic modifications in cultured hippocampal neurons: dependence on spike timing, synaptic strength, and postsynaptic cell type. J. Neurosci. 18, 10464 (1998).

15. Whitlock, J. R., Heynen, A. J., Shouler, M. G. \& Bear, M. F. Learning induces long-term potentiation in the hippocampus. Science 313, 1093-1097 (2006).

16. Takeo, O. et al. Short-term plasticity and long-term potentiation mimicked in single inorganic synapses. Nat. Mater. 3054, 591-595 (2011).

17. Burr, G. W. et al. Experimental demonstration and tolerancing of a large-scale neural network (165,000 synapses) using phase-change memory as the synaptic weight element. IEEE Trans. 62, 3498-3507 (2015).

18. Yang, J. J., Strukov, D. B. \& Stewart, D. R. Memristive devices for computing Nat. Nanotech. 8, 13-24 (2013).

19. Chen, P. Y., Peng, X. \& Yu, S. NeuroSim+: an integrated device-to-algorithm framework for benchmarking synaptic devices and array architectures. In 2017 IEEE International Electron Devices Meeting (IEDM) 135-138 (IEEE, San Francisco, 2017)

20. Yu, S. Neuro-inspired computing with emerging nonvolatile memory. Proc. IEEE 106, 260-285 (2018) 
21. Laughltn, S. B., de Ruyter van Steveninck, R. R. \& Anderson, J. C. The metabolic cost of neural information. Nat. Neurosci. 1, 36-41 (1998).

22. Shim, J. et al. Electronic and optoelectronic devices based on two-dimensional materials: from fabrication to application. Adv. Electron. Mat. 3, 1600364 (2017).

23. Geim, A. K. \& Grigorieva, I. V. Van der Waals heterostructures. Nature 499, 419-425 (2013).

24. Lemme, M. C., Li, L.-J., Palacios, T. \& Schwierz, F. Two-dimensional materials for electronic applications. Mrs. Bull. 39, 711-718 (2014).

25. Jo, S.-H. et al. A high-performance WSe2/h-BN photodetector using a triphenylphosphine (PPh3)-based n-doping technique. Adv. Mat. 28, 4824-4831 (2016).

26. Mochida, R. et al. A 4M synapses integrated analog ReRAM based 66.5 TOPS/ W neural network processor with Cell Current Writing and Flexible Network Architecture. In 2018 Symposium on VLSI Technology (IEEE, Honolulu, 2018).

27. Ambrogio. et al. Equivalent-accuracy accelerated neural-entwork training using analogue memory. Nature 558, 60-67 (2018).

28. Querlioz, D., Bichler, O., Dollfus, P. \& Gamrat, C. Immunity to device variations in a spiking neural network with memristive nanodevices. IEEE Trans. 12, 288-295 (2013)

29. Anold, A. J. et al. Mimicking neurotransmitter release in chemical synapses via hysteresis engineering in $\mathrm{MoS}_{2}$ transistors. ACS Nano 11, 3310-3118 (2017).

30. Rumelhart, D. E., Hinton, G. E., \& Willians, R. J. in Parallel Distributed Processing: Explorations in Macrostructure of Cognition. Vol. 1 (eds Rumelhart, D. E. \& McClelland, J. L.) 318-362 (MIT Press, Cambridge,1986).

31. Lim, S. et al. Adaptive learning rule for hardware-based deep neural networks using electronic synapse devices. Preprint available at http://arXiv.org/abs/ 1707.06381 (2017).

32. Han, S., Mao, H. \& Dally, W. J. Deep compression: compressing deep neural networks with pruning, trained quantization and Huffman coding. In International Conference on Learning Representations 2016 (ICLR, 2016).

33. Moons, B. \& Verhelst, M. A 0.3-2.6 TOPS/W Precision-Scalable Processor for Real-Time Large-Scale ConvNets. In 2016 Symposium on VLSI Circuits (IEEE, Honolulu, ICLR, San Juan, 2016).

34. Dan, Y. \& Poo, M.-M. Spike timing-dependent plasticity: from synapse to perception. Phy. Rev. 86, 1033-1048 (2006).

35. Hertz, J., Krogh, A. \& Palmer, R. G. Introduction to the Theory of Neural Computation (Perseus, Cambridge, 1991).

36. Lecun, Y., Cortes, C. \& Burges, C. J. C. Gradient-based learning applied to document recognition. Proc. IEEE 86, 2278-2324 (1998).

\section{Acknowledgements}

This research was supported by the Basic Research Lab Program and Basic Science Research Program through the National Research Foundation of Korea (NRF) funded by the Ministry of Science, ICT (2017R1A4A1015400, 2018R1A2A2A05020475), and the Nano-Material Technology Development Program through the National Research Foundation of Korea (NRF) funded by the Ministry of Science, ICT (2016M3A7B4910426).

\section{Author contributions}

S.S., J.S.-H., K.S., C.C., O.S., K.D., W.H.-S. P. and P.J.-H. designed the experiments and analyzed the data. S.J. and C.J.-W. contributed to the device fabrication. H.K. performed the X-TEM and EELS/EDS measurements. O.S. and K.J.-H. performed the pattern recognition simulation. J.-H.P. supervised the research. All authors have discussed the results and commented on the manuscript.

\section{Additional information}

Supplementary Information accompanies this paper at https://doi.org/10.1038/s41467018-07572-5.

Competing interests: The authors declare no competing interests.

Reprints and permission information is available online at http://npg.nature.com/ reprintsandpermissions/

Publisher's note: Springer Nature remains neutral with regard to jurisdictional claims in published maps and institutional affiliations.

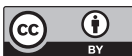

Open Access This article is licensed under a Creative Commons Attribution 4.0 International License, which permits use, sharing adaptation, distribution and reproduction in any medium or format, as long as you give appropriate credit to the original author(s) and the source, provide a link to the Creative Commons license, and indicate if changes were made. The images or other third party material in this article are included in the article's Creative Commons license, unles indicated otherwise in a credit line to the material. If material is not included in the article's Creative Commons license and your intended use is not permitted by statutory regulation or exceeds the permitted use, you will need to obtain permission directly from the copyright holder. To view a copy of this license, visit http://creativecommons.org/ licenses/by/4.0/.

(C) The Author(s) 2018 